\title{
STABILITY AND BIFURCATION OF NUMERICAL DISCRETIZATION NICHOLSON BLOWFLIES EQUATION WITH DELAY
}

\author{
XIAOHUA DING AND WENXUE LI
}

Received 31 December 2005; Accepted 13 March 2006

A kind of discrete system according to Nicholson's blowflies equation with a finite delay is obtained by the Euler forward method, and the dynamics of this discrete system are investigated. Applying the theory of normal form and center manifold, we not only discuss the linear stability of the equilibrium and the existence of the local Hopf bifurcations, but also give the explicit algorithm for determining the direction of bifurcation and stability of the periodic solution of bifurcation.

Copyright (c) 2006 X. Ding and W. Li. This is an open access article distributed under the Creative Commons Attribution License, which permits unrestricted use, distribution, and reproduction in any medium, provided the original work is properly cited.

\section{Introduction}

Equation

$$
x^{\prime}(t)=a x(t-\tau) e^{-b x(t-\tau)}-c x(t)
$$

which is one of the most important ecological systems, describes the population dynamics of Nicholson's blowflies. Here $x(t)$ is the size of the population at time $t, a$ is the maximum per capita daily egg production rate, $1 / b$ is the size at which the population reproduces at the maximum rate, $c$ is the per capita daily adult death rate, and $\tau$ is the generation time. Equation (1.1) has been extensively studied in the literature [2, 7]. The majority of the results on (1.1) deal with the global attractiveness of the positive equilibrium and oscillatory behaviors of solutions $[1,3-6,9]$.

But in practice, we need computers to simulate system (1.1), so it is necessary to study the discrete system of (1.1). We hope that the dynamic behaviors of the discrete system accord with the ones of system (1.1). Wei and Li [10] have got the condition upon which a Hopf bifurcation exists and the bifurcating periodic solutions are stable for (1.1). In this paper, we study the dynamics behaviors of the discrete system of (1.1), such as the existence of Hopf bifurcation and the stability of the bifurcating periodic solution. The theoretical analysis gives the condition under which there exists a Hopf bifurcation and 


\section{Stability and bifurcation of discrete system}

the bifurcating periodic solutions are stable for the discrete system. At last the numerical test shows that the analytic results are correct.

The paper is organized as follows: in Section 2, we investigate the occurrence of Hopf bifurcations. In Section 3, direction and stability of the Hopf bifurcation of discrete model are established. In Section 4, computer simulations are performed to illustrate the analytical results found.

\section{Stability analysis}

Let $u(t)=x(\tau t)$. Then (1.1) can be written as

$$
u^{\prime}(t)=a \tau u(t-1) e^{-b u(t-1)}-c \tau u(t) .
$$

We consider the stepsize of the form $h=1 / m$, where $m \in \mathbb{Z}^{+}$. Applying Euler method to this equation yields the difference equation

$$
u_{n+1}=(1-c \tau h) u_{n}+a \tau h u_{n-m} e^{-b u_{n-m}},
$$

where $u_{n}$ is an approximate value to $u(n h)$.

Set $u^{*}$ a positive fixed point of (2.2), then we have

$$
c=a e^{-b u^{*}} .
$$

Substituting $y_{n}=u_{n}-u^{*}$ into (2.2) deduces that

$$
y_{n+1}=(1-c \tau h) y_{n}+c \tau h\left(y_{n-m}+u^{*}\right) e^{-b y_{n-m}}-c \tau h u^{*} .
$$

Introducing a new variable, $Y_{n}=\left(y_{n}, y_{n-1}, \ldots, y_{n-m}\right)^{T}$, we can rewrite (2.4) as

$$
Y_{n+1}=F\left(Y_{n}, \tau\right) \text {, }
$$

where $F=\left(F_{0}, F_{1}, \ldots, F_{m}\right)^{T}$ and

$$
F_{k}= \begin{cases}(1-c \tau h) y_{n}+c \tau h\left(y_{n-m}+u^{*}\right) e^{-b y_{n-m}}-c \tau h u^{*}, & k=0 ; \\ y_{n-k+1}, & 1 \leq k \leq m .\end{cases}
$$

Clearly the origin is a fixed point of map (2.5), the linear part of map (2.5) is

$$
Y_{n+1}=A Y_{n}
$$

where

$$
A=\left(\begin{array}{ccccc}
1-c \tau h & 0 & \cdots & 0 & c \tau h\left(1-b u^{*}\right) \\
1 & 0 & \cdots & 0 & 0 \\
0 & 1 & \cdots & 0 & 0 \\
\vdots & \vdots & \vdots & \vdots & \vdots \\
0 & 0 & \cdots & 1 & 0
\end{array}\right)
$$


It is easy to see that the characteristic equation of $A$ is

$$
z^{m}(z-1+c \tau h)-c \tau h\left(1-b u^{*}\right)=0 .
$$

It is well known that the stability of the fixed point of map (2.5) depends on the distribution of the zeros of (2.9).

We will employ the results from Ruan and Wei [8] and Zhang et al. [12] to analyze the distribution of the roots of the characteristic (2.9).

Lemma 2.1 [12]. Suppose that $B \in \mathbb{R}$ is a bounded closed and connected set, $f(\lambda, \tau)=\lambda^{m}+$ $p_{1}(\tau) \lambda^{m-1}+p_{2}(\tau) \lambda^{m-2}+\cdots+p_{m}(\tau)$ is continuous in $(\lambda, \tau) \in \mathbb{C} \times B$, and $\tau$ is a parameter, $\tau \in B$. Then as $\tau$ varies, the sum of the order of the zeros of $f(\lambda, \tau)$ out of the unit circle

$$
\{\lambda \in \mathbb{C}:|\lambda|>1\}
$$

can change only if a zero appears on or crosses the unit circle.

Lemma 2.2. There exists $\tau^{\prime}>0$ such that for $0<\tau<\tau^{\prime}$ all roots of (2.9) have modulus less than one.

Proof. When $\tau=0$, (2.9) becomes

$$
z^{m+1}-z^{m}=0
$$

The equation has, as $\tau=0$, an $m$-fold root and a simple root $z=1$.

Consider the root $z(\tau)$ such that $|z(0)|=1$. This root depends continuously on $\tau$ and is a differential function of $\tau$. From (2.9), we have

$$
\begin{aligned}
& \frac{d z}{d \tau}=\frac{c h\left(1-b u^{*}\right)-c h z^{m}}{m z^{m+1}(z-1+c \tau h)+z^{m}}, \\
& \frac{d \bar{z}}{d \tau}=\frac{c h\left(1-b u^{*}\right)-c h \bar{z}^{m}}{m \bar{z}^{m+1}(\bar{z}-1+c \tau h)+\bar{z}^{m}},
\end{aligned}
$$

so

$$
\begin{gathered}
\frac{d|z|^{2}}{d \tau}=z \frac{d \bar{z}}{d \tau}+\bar{z} \frac{d z}{d \tau} \\
\left.\frac{d|z|^{2}}{d \tau}\right|_{\tau=0, z=1}=-2 b c h u^{*}<0 .
\end{gathered}
$$

Consequently, $|z|<1$ for all sufficiently small $\tau>0$. Thus all roots of (2.9) lie in $|z|<1$ for sufficiently small positive $\tau>0$, and the existence of the maximal $\tau^{\prime}$ follows.

Lemma 2.3. Assume that the stepsize $h$ is sufficiently small and $b u^{*}<2$. Then (2.9) has no root with modulus one for all $\tau>0$.

Proof. Let $e^{i \omega^{*}}$ be a root of (2.9) when $\tau=\tau^{*}$. Then

$$
e^{i(m+1) \omega^{*}}-e^{i m \omega^{*}}(1-c \tau h)=c \tau h\left(1-b u^{*}\right) .
$$


4 Stability and bifurcation of discrete system

Separating the real and imaginary parts, we have

$$
\begin{gathered}
\cos (m+1) \omega^{*}-(1-c \tau h) \cos m \omega^{*}=c \tau h\left(1-b u^{*}\right), \\
\sin (m+1) \omega^{*}-(1-c \tau h) \sin m \omega^{*}=0
\end{gathered}
$$

so

$$
\begin{aligned}
\cos \omega^{*} & =\frac{1-(c \tau h)^{2}\left(1-b u^{*}\right)^{2}+(1-c \tau h)^{2}}{2(1-c \tau h)} \\
& =1+\frac{(c \tau h)^{2} b u^{*}\left(2-b u^{*}\right)}{2(1-c \tau h)}
\end{aligned}
$$

For $h>0$ small enough, if $b u^{*}<2$, then $\cos \omega^{*}>1$, which yields a contradiction. This completes the proof.

If $b u^{*}>2$, then the roots $e^{ \pm i \omega^{*}}$ of (2.9) with modulus one satisfy

$$
\begin{gathered}
\cos \omega^{*}=1+\frac{(c \tau h)^{2} b u^{*}\left(2-b u^{*}\right)}{2(1-c \tau h)}, \\
\tau^{*}=\frac{1}{c h}\left(1-\frac{\sin (m+1) \omega^{*}}{\sin m \omega^{*}}\right), \\
h=\frac{1}{m} .
\end{gathered}
$$

Lemma 2.4. If the stepsize $h$ is sufficiently small and $b u^{*}>2$, then

$$
d_{h}=\left.\frac{d|z|^{2}}{d \tau}\right|_{\tau=\tau^{*}, \omega=\omega^{*}}>0,
$$

where $\tau^{*}$ and $\omega^{*}$ satisfy (2.17).

Proof. From (2.9), we have

$$
\begin{aligned}
d_{h} & =\left.\left[z \frac{d \bar{z}}{d \tau}+\bar{z} \frac{d z}{d \tau}\right]\right|_{\tau=\tau^{*}, \omega=\omega^{*}} \\
& =\left.\left[\bar{z} \frac{c h\left(1-b u^{*}\right)-c h z^{m}}{m z^{m+1}(z-1+c \tau h)+z^{m}}+z \frac{c h\left(1-b u^{*}\right)-c h \bar{z}^{m}}{m \bar{z}^{m+1}(\bar{z}-1+c \tau h)+\bar{z}^{m}}\right]\right|_{\tau=\tau^{*}, \omega=\omega^{*}} \\
& =2 \operatorname{ch} \frac{(m+1)\left[\left(1-b u^{*}\right) \cos (m+1) \omega^{*}-\cos \omega^{*}\right]+m(1-c \tau h)\left[1-\left(1-b u^{*}\right) \cos m \omega^{*}\right]}{(m+1)^{2}+m^{2}(1-c \tau h)^{2}-2 m(m+1)(1-c \tau h) \cos \omega^{*}} .
\end{aligned}
$$

By (2.15) we find that

$$
\cos m \omega^{*}=\frac{1}{1-b u^{*}}+\frac{c \tau h b u^{*}\left(2-b u^{*}\right)}{2(1-c \tau h)\left(1-b u^{*}\right)},
$$


so we derive that

$$
\begin{aligned}
\cos (m+1) \omega^{*} & =c \tau h\left(1-b u^{*}\right)+(1-c \tau h) \cos m \omega^{*} \\
& =c \tau h\left(1-b u^{*}\right)+\frac{1-c \tau h}{1-b u^{*}}+\frac{c \tau h b u^{*}\left(2-b u^{*}\right)}{2\left(1-b u^{*}\right)}
\end{aligned}
$$

It is easy to see that

$$
\begin{gathered}
1-\left(1-b u^{*}\right) \cos m \omega^{*}=-\frac{c \tau h b u^{*}\left(2-b u^{*}\right)}{2(1-c \tau h)}>0, \\
\left(1-b u^{*}\right) \cos (m+1) \omega^{*}-\cos \omega^{*}=\frac{1}{2} b u^{*}\left(b u^{*}-2\right)\left(1-\frac{c \tau h}{1-c \tau h}\right)>0 .
\end{gathered}
$$

Equation (2.22) implies immediately that

$$
d_{h}=\left.\frac{d|z|^{2}}{d \tau}\right|_{\tau=\tau^{*}, \omega=\omega^{*}}>0
$$

hold. This completes the proof.

By Lemmas 2.1-2.4, we have the following results on stability and bifurcation in map (2.5).

Theorem 2.5. (1) If bu* $<2$, then the fixed point of map (2.5) is absolutely stable for all $\tau>0$.

(2) If $b u^{*}>2$, then there exists an infinite sequence of the time delay parameter, $\tau_{0}<$ $\tau_{1}<\cdots<\tau_{n}<\cdots$, such that the fixed point of map (2.5) is asymptotically stable when $\tau \in\left(0, \tau_{0}\right)$ and unstable when $\tau>\tau_{0}$. Map (2.5) has a Hopf bifurcation at the origin when $\tau=\tau_{j}, j=0,1,2 \ldots$, where $\tau_{j}$ satisfies (2.17).

Proof. (1) Set $b u^{*}<2$. From Lemmas 2.2 and 2.3, we know that (2.9) has no root with modulus one for all $\tau>0$. Applying Lemma 2.1, all roots of (2.9) have modulus less than one for all $\tau>0$. Hence, conclusion (1) follows.

(2) Set $b u^{*}>2$. Applying Lemma 2.4, we know that all roots of (2.9) have modulus less than one when $\tau \in\left(0, \tau_{0}\right)$, and (2.9) has at least a couple of roots with modulus greater than one when $\tau>\tau_{0}$. Hence conclusion (2) follows.

\section{Direction and stability of the Hopf bifurcation in discrete model}

Without loss of generality, denote the critical value $\tau_{j}(j=0,1,2, \ldots)$ by $\tau^{*}$, at which map (2.5) undergoes a Hopf bifurcation at origin. Assume map (2.5) is smooth enough so that it can be expanded.

For map (2.5), we have

$$
Y_{n+1}=A Y_{n}+\frac{1}{2} B\left(Y_{n}, Y_{n}\right)+\frac{1}{6} \mathbb{C}\left(Y_{n}, Y_{n}, Y_{n}\right)+O\left(\left\|Y_{n}\right\|^{4}\right)
$$


6 Stability and bifurcation of discrete system

where

$$
\begin{gathered}
B\left(Y_{n}, Y_{n}\right)=\left(b_{0}\left(Y_{n}, Y_{n}\right), 0 \cdots 0\right), \\
\mathbb{C}\left(Y_{n}, Y_{n}, Y_{n}\right)=\left(c_{0}\left(Y_{n}, Y_{n}, Y_{n}\right), 0 \cdots 0\right), \\
b_{0}(\phi, \psi)=c \tau h b\left(b u^{*}-2\right) \phi_{m} \psi_{m}, \\
c_{0}(\phi, \psi, \eta)=c \tau h b^{2}\left(3-b u^{*}\right) \phi_{m} \psi_{m} \eta_{m} .
\end{gathered}
$$

Let $q \in \mathbb{C}^{m+1}$ be a complex eigenvector of $A$ corresponding to $e^{i \omega^{*}}$, then

$$
A q=e^{i \omega^{*}} q, \quad A \bar{q}=e^{-i \omega^{*}} \bar{q} .
$$

We also introduce an adjoint eigenvector $q^{*} \in \mathbb{C}^{m+1}$ having the properties

$$
A^{T} q^{*}=e^{-i \omega^{*}} q^{*}, \quad A^{T} \bar{q}^{*}=e^{i \omega^{*}} \bar{q}^{*}
$$

and satisfying the normalization $\left\langle q^{*}, q\right\rangle=1$, where $\left\langle q^{*}, q>=\sum_{i=0}^{m} \bar{q}_{i}^{*} q_{i}\right.$.

Lemma 3.1. Let $q=\left(q_{0}, q_{1}, \ldots, q_{m}\right)^{T}$ be the eigenvector of $A$ corresponding to the eigenvalue $e^{i \omega^{*}}$, and $q^{*}=\left(q_{0}^{*}, q_{1}^{*}, \ldots, q_{m}^{*}\right)$ be the eigenvector of $A^{T}$ corresponding to the eigenvalue $e^{-i \omega^{*}}$. Then

$$
\begin{aligned}
q & =\left(1, e^{-i \omega^{*}}, \ldots, e^{-i m \omega^{*}}\right)^{T}, \\
q^{*} & =K\left(1, \alpha e^{i m \omega^{*}}, \ldots, \alpha e^{i \omega^{*}}\right),
\end{aligned}
$$

where $\alpha=c \tau h\left(1-b u^{*}\right)$ and $K=\left(1+c \tau h\left(1-b u^{*}\right) m e^{i(m+1) \omega^{*}}\right)^{-1}$.

Proof. Let $q=\left(q_{0}, q_{1}, \ldots, q_{m}\right)^{T}$ be the eigenvector of $A$ corresponding to the eigenvalue $e^{i \omega^{*}}$, then

$$
\begin{gathered}
q_{i}=q_{i+1} e^{i \omega^{*}}, \quad i=1,2, \ldots, m-1, \\
(1-c \tau h) q_{0}+\tau h\left(1-b u^{*}\right) q_{m}=q_{0} e^{i \omega^{*}} .
\end{gathered}
$$

Setting $q_{0}=1$ we find that

$$
q=\left(1, e^{-i \omega^{*}}, \ldots, e^{-i m \omega^{*}}\right)^{T}
$$

is the eigenvector of $A$ corresponding to the eigenvalue $e^{i \omega^{*}}$. Similarly,

$$
q^{*}=K\left(1, \alpha e^{i m \omega^{*}}, \ldots, \alpha e^{i \omega^{*}}\right),
$$

where $\alpha=c \tau h\left(1-b u^{*}\right)$ and $K=\left(1+c \tau h\left(1-b u^{*}\right) m e^{i(m+1) \omega^{*}}\right)^{-1}$.

All vectors $x \in \mathbb{R}^{m+1}$ can be decomposed as

$$
x=v q+\bar{v} \bar{q}+y
$$




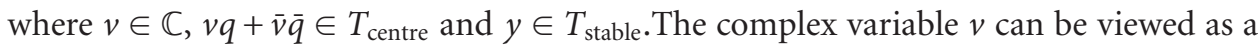
new coordinate on $T_{\text {centre }}$, so we have

$$
\begin{gathered}
v=\left\langle q^{*}, x\right\rangle, \\
y=x-\left\langle q^{*}, x\right\rangle q-\left\langle\bar{q}^{*}, x\right\rangle \bar{q} .
\end{gathered}
$$

Let $a(\mu)$ be a characteristic polynomial of $A$ and $\mu=\mu(\tau)$ be a characteristic root of $A$. Applying this decomposition to the map $F$, we get

$$
\begin{gathered}
v \longmapsto \mu(\tau) v+\left\langle q^{*}, N(v q+\bar{v} \bar{q}+y)\right\rangle, \\
y \longmapsto A y+N(v q+\bar{v} \bar{q}+y)-\left\langle q^{*}, N(v q+\bar{v} \bar{q}+y)\right\rangle q-\left\langle\bar{q}^{*}, N(v q+\bar{v} \bar{q}+y)\right\rangle \bar{q} .
\end{gathered}
$$

Using Taylor expansions, we get

$$
v \longmapsto \mu(\tau) v+\frac{1}{2} g_{20} v^{2}+g_{11} v \bar{v}+\frac{1}{2} g_{02} \bar{v}^{2}+\frac{1}{2} g_{21} v^{2} \bar{v}+\cdots
$$

Let $\tau=\tau^{*}$, then we obtain

$$
v \longmapsto e^{i \omega^{*}} v+\frac{1}{2} g_{20} v^{2}+g_{11} v \bar{v}+\frac{1}{2} g_{02} \bar{v}^{2}+\frac{1}{2} g_{21} v^{2} \bar{v}+\cdots,
$$

where

$$
\begin{gathered}
g_{20}=\left\langle q^{*}, B(q, q)\right\rangle, \\
g_{11}=\left\langle q^{*}, B(q, \bar{q})\right\rangle, g_{02}=\left\langle q^{*}, B(\bar{q}, \bar{q})\right\rangle, \\
g_{21}=\left\langle q^{*}, B\left(\bar{q}, \omega_{20}\right)\right\rangle+2\left\langle q^{*}, B\left(q, \omega_{11}\right)\right\rangle+\left\langle q^{*}, \mathbb{C}(q, q, \bar{q})\right\rangle, \\
\left(e^{i \omega^{*}} I-A\right) \omega_{20}=H_{20}, \\
(I-A) \omega_{11}=H_{11}, \\
H_{20}=B_{q, q}-\left\langle q^{*}, B(q, q)\right\rangle q-\left\langle\bar{q}^{*}, B(q, q)\right\rangle \bar{q}, \\
H_{11}=B_{q, \bar{q}}-\left\langle q^{*}, B(q, \bar{q})\right\rangle q-\left\langle\bar{q}^{*}, B(q, \bar{q})\right\rangle \bar{q} .
\end{gathered}
$$

By [11, Lemmas 3.8 and 3.10], we get

$$
\begin{aligned}
& \omega_{20}=\frac{b_{0}(q, q)}{a\left(\mu^{2}\right)} q\left(\mu^{2}\right)-\frac{\left\langle q^{*}, B(q, q)\right\rangle}{\mu^{2}-\mu} q-\frac{\left\langle\bar{q}^{*}, B(q, q)\right\rangle}{\mu^{2}-\bar{\mu}} \bar{q}, \\
& \omega_{11}=\frac{b_{0}(q, \bar{q})}{a(1)} q(1)-\frac{\left\langle q^{*}, B(q, \bar{q})\right\rangle}{1-\mu} q-\frac{\left\langle\bar{q}^{*}, B(q, \bar{q})\right\rangle}{1-\bar{\mu}} \bar{q},
\end{aligned}
$$


8 Stability and bifurcation of discrete system

here $\mu=e^{i \omega^{*}}$ is a characteristic root of $A$. The coefficient $g_{21}$ can be simplified as

$$
\begin{aligned}
g_{21}= & \frac{b_{0}(q, \bar{q})}{a\left(\mu^{2}\right)}\left\langle q^{*}, B\left(\bar{q}, q\left(\mu^{2}\right)\right)\right\rangle-\frac{g_{20} g_{11}}{\mu^{2}-\mu}-\frac{\left|g_{02}\right|^{2}}{\mu_{2}-\mu} \\
& +2 \frac{b_{0}(q, \bar{q})}{a(1)}\left\langle q^{*}, B(q, q(1))\right\rangle-2 \frac{g_{20} g_{11}}{1-\mu}-2 \frac{\left|g_{11}\right|^{2}}{1-\bar{\mu}}+\left\langle q^{*}, \mathbb{C}(q, q, \bar{q})\right\rangle .
\end{aligned}
$$

From this we obtain an expression for the critical coefficient $c_{1}$ :

$$
c_{1}=\frac{1}{2} \bar{q}_{0}^{*}\left[\frac{b_{0}\left(\bar{q}, q\left(\mu^{2}\right)\right) b_{0}(q, q)}{a\left(\mu^{2}\right)}+\frac{2 b_{0}(q, q(1)) b_{0}(q, \bar{q})}{a(1)}+c_{0}(q, q, \bar{q})\right] .
$$

Lemma 3.2 [11]. Given the map (3.13), assume the following.

(1) $\mu(\tau)=r(\tau) e^{i \omega(\tau)}, \mu(\tau), \omega(\tau) \in \mathbb{R}$, where

$$
r\left(\tau^{*}\right)=1, \quad r^{\prime}\left(\tau^{*}\right) \neq 0, \quad \omega\left(\tau^{*}\right)=\omega^{*}
$$

(2) $e^{i k \omega^{*}} \neq 1$ for $k=1,2,3,4$.

(3) Let

$$
c_{1}=\frac{1}{2} \bar{q}_{0}^{*}\left[\frac{b_{0}\left(\bar{q}, q\left(\mu^{2}\right)\right) b_{0}(q, q)}{a\left(\mu^{2}\right)}+\frac{2 b_{0}(q, q(1)) b_{0}(q, \bar{q})}{a(1)}+c_{0}(q, q, \bar{q})\right],
$$

where $\mu=\mu\left(\tau^{*}\right)=e^{i \omega^{*}}$ and the $g_{i, j}$ are evaluated at $\tau=\tau^{*}$, and assume

$$
\operatorname{Re}\left[e^{-i \omega^{*}} c_{1}\left(\tau^{*}\right)\right] \neq 0 \text {. }
$$

Then an invariant closed curve, topologically equivalent to a circle, for the map exists for $\tau$ in a one side neighborhood of $\tau^{*}$. The radius of the invariant curve grows like $O\left(\sqrt{\left|\tau-\tau^{*}\right|}\right)$. One of the four cases below applies:

(1) $r^{\prime}\left(\tau^{*}\right)>0, \operatorname{Re}\left[e^{-i \omega^{*}} c_{1}\left(\tau^{*}\right)\right]<0$. The origin is asymptotically stable for $\tau<\tau^{*}$ and unstable for $\tau>\tau^{*}$. An attracting invariant closed curve exists for $\tau>\tau^{*}$;

(2) $r^{\prime}\left(\tau^{*}\right)>0, \operatorname{Re}\left[e^{-i \omega^{*}} c_{1}\left(\tau^{*}\right)\right]>0$. The origin is asymptotically stable for $\tau<\tau^{*}$ and unstable for $\tau>\tau^{*}$. A repelling invariant closed curve exists for $\tau<\tau^{*}$;

(3) $r^{\prime}\left(\tau^{*}\right)<0, \operatorname{Re}\left[e^{-i \omega^{*}} c_{1}\left(\tau^{*}\right)\right]<0$. The origin is asymptotically stable for $\tau>\tau^{*}$ and unstable for $\tau<\tau^{*}$. An attracting invariant closed curve exists for $\tau<\tau^{*}$;

(4) $r^{\prime}\left(\tau^{*}\right)<0, \operatorname{Re}\left[e^{-i \omega^{*}} c_{1}\left(\tau^{*}\right)\right]>0$. The origin is asymptotically stable for $\tau>\tau^{*}$ and unstable for $\tau<\tau^{*}$. An attracting invariant closed curve exists for $\tau>\tau^{*}$.

TheOREm 3.3. If $b u^{*}>2$ holds, then the origin is asymptotically stable for $\tau<\tau^{*}$ and unstable for $\tau>\tau^{*}$. An attracting invariant closed curve exists for $\tau>\tau^{*}$. 
Proof. By (3.3), we know

$$
\begin{gathered}
b_{0}\left(\bar{q}, q\left(\mu^{2}\right)\right)=c \tau^{*} h b\left(b u^{*}-2\right) e^{-i m \omega^{*},} \\
b_{0}(q, q)=c \tau^{*} h b\left(b u^{*}-2\right) e^{-i m \omega^{*},} \\
b_{0}(q, \bar{q})=c \tau^{*} h b\left(b u^{*}-2\right), \\
c_{0}(q, q, \bar{q})=c \tau^{*} h b^{2}\left(3-b u^{*}\right) e^{-i m \omega^{*},} \\
a\left(\mu^{2}\right)=e^{i(2 m+2) \omega^{*}}-c \tau^{*} h b\left(1-u^{*}\right)-c \tau^{*} h e^{i 2 m \omega^{*},} \\
a(1)=1-c \tau^{*} h b\left(b u^{*}-2\right), \\
b_{0}(q, q(1))=c \tau^{*} h b\left(b u^{*}-2\right) e^{-i m \omega^{*}} .
\end{gathered}
$$

By (3.8), (3.9), (3.18), and (3.22) we get

$$
\begin{array}{r}
c_{1}\left(\tau^{*}\right)=\frac{\bar{k} c \tau^{*} h b e^{-i m \omega^{*}}}{2}\left(\frac{c \tau^{*} h b\left(b u^{*}-2\right)^{2} e^{-i 2 m \omega^{*}}}{e^{i(2 m+2) \omega^{*}}-c \tau^{*} h b\left(1-u^{*}\right)-c \tau^{*} h e^{i 2 m \omega^{*}}}\right. \\
\left.+\frac{c \tau^{*} h b\left(b u^{*}-2\right)^{2}}{1-c \tau^{*} h b\left(b u^{*}-2\right)}+b\left(3-b u^{*}\right)\right) .
\end{array}
$$

So, by (2.15) (2.16), and (2.20) we have

$$
\operatorname{Re}\left[e^{-i \omega^{*}} c_{1}\left(\tau^{*}\right)\right]=-\frac{1-b u^{*}\left(2-b u^{*}\right) h}{2 \triangle\left(b u^{*}-1\right)^{2}}+O\left(h^{2}\right),
$$

where

$$
\triangle=\left|e^{-2 i(m+1) \omega^{*}}-c \tau^{*} h-c h m \tau^{*} e^{-2 i m \omega^{*}}+b c h \tau^{*} u^{*}\right|^{2}\left|1+c h m \tau^{*} e^{-2 i(m+1) \omega^{*}}\left(1-b u^{*}\right)\right|^{2} .
$$

so

$$
\operatorname{Re}\left[e^{-i \omega^{*}} c_{1}\left(\tau^{*}\right)\right]<0,
$$

where $\tau=\tau^{*}, \omega=\omega^{*}$ satisfy (2.17). By lemmas 2.4 and 3.2, the theorem as follows.

\section{Numerical test}

To illustrate the analytical results found, let us consider the following particular case of system (2.2). We could get that $a=30, b=2, c=2$, then $\tau \approx 1.1994$ is the Hopf bifurcation value and $b u^{*} \approx 2.708>2$. 


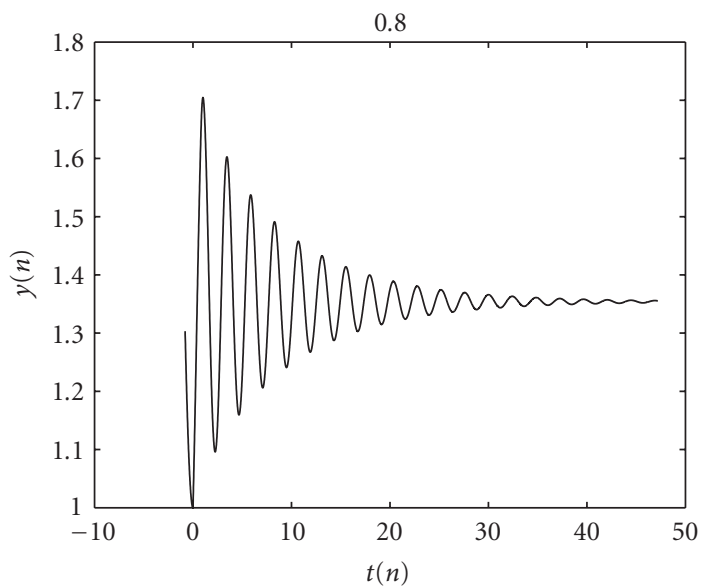

Figure 4.1. The equilibrium of (2.2) is asymptotically stable when $\tau=0.8$.

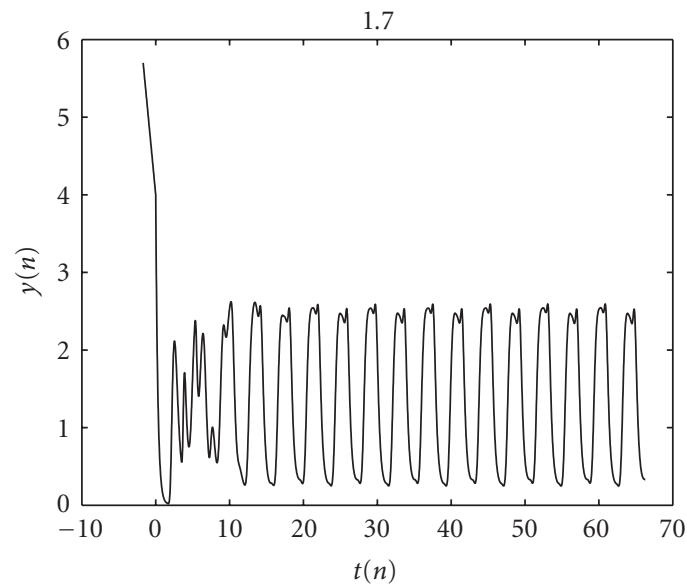

Figure 4.2. The equilibrium of (2.2) is unstable when $\tau=1.7$.

Figures 4.1, 4.2, and 4.3 are about difference equation (2.2) when the stepsize $h=$ $\tau / 100$.

From Figure 4.1, $\tau=0.8$, we could get that the equilibrium $u^{*}=1.354$ of (2.2) is asymptotically stable. And from Figures 4.2 and 4.3, $\tau=1.7$ and 1.3, we have the equilibrium $u^{*}=1.354$ of (2.2) is unstable; the system undergoes a periodic solution of bifurcation. 


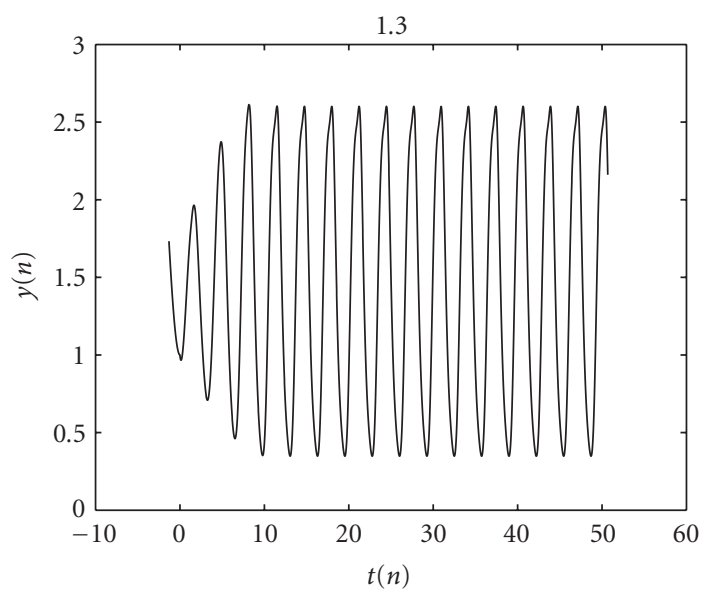

Figure 4.3. System exists on attracting invariant closed curve for $\tau=1.3>1.1994$.

\section{Acknowledgment}

This work is supported by the NSF of P. R. China (no. 10271036) and of HIT (200518).

\section{References}

[1] Q. X. Feng and J. R. Yan, Global attractivity and oscillation in a kind of Nicholson's blowflies, Journal of Biomathematics 17 (2002), no. 1, 21-26.

[2] I. Györi and G. Ladas, Oscillation Theory of Delay Differential Equations. With Applications, Oxford Mathematical Monographs, Oxford University Press, Oxford, 1991.

[3] M. R. S. Kulenović and G. Ladas, Linearized oscillations in population dynamics, Bulletin of Mathematical Biology 49 (1987), no. 5, 615-627.

[4] M. R. S. Kulenović, G. Ladas, and Y. G. Sficas, Global attractivity in population dynamics, Computers \& Mathematics with Applications 18 (1989), no. 10-11, 925-928.

[5] J. Li, Global attractivity in a discrete model of Nicholson's blowflies, Annals of Differential Equations 12 (1996), no. 2, 173-182.

[6] __ Global attractivity in Nicholson's blowflies, Applied Mathematics. Series B 11 (1996), no. $4,425-434$.

[7] M. Li and J. Yan, Oscillation and global attractivity of generalized Nicholson's blowfly model, Differential Equations and Computational Simulations (Chengdu, 1999), World Scientific, New Jersey, 2000, pp. 196-201.

[8] S. Ruan and J. Wei, On the zeros of transcendental functions with applications to stability of delay differential equations with two delays, Dynamics of Continuous, Discrete \& Impulsive Systems. Series A. Mathematical Analysis 10 (2003), no. 6, 863-874.

[9] J. W.-H. So and J. S. Yu, On the stability and uniform persistence of a discrete model of Nicholson's blowflies, Journal of Mathematical Analysis and Applications 193 (1995), no. 1, 233-244.

[10] J. Wei and M. Y. Li, Hopf bifurcation analysis in a delayed Nicholson blowflies equation, Nonlinear Analysis 60 (2005), no. 7, 1351-1367.

[11] V. Wulf, Numerical Analysis of Delay Differential Equations Undergoing a Hopf Bifurcation, University of Liverpool, 1999. 
12 Stability and bifurcation of discrete system

[12] C. Zhang, Y. Zu, and B. Zheng, Stability and bifurcation of a discrete red blood cell survival model, Chaos Solitons and Fractals 28 (2006), no. 2, 386-394.

Xiaohua Ding: Department of Mathematics, Harbin Institute of Technology (Weihai),

Weihai 264209, China

E-mail address: mathdxh@126.com

Wenxue Li: Department of Mathematics, Harbin Institute of Technology (Weihai), Weihai 264209, China

E-mail address:wenxue810823@163.com 


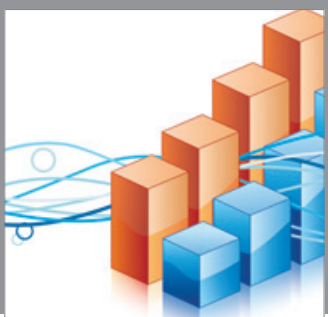

Advances in

Operations Research

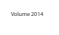

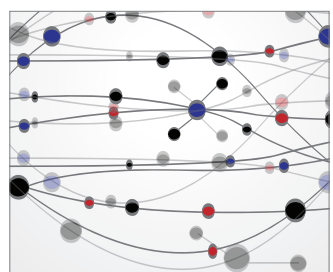

\section{The Scientific} World Journal
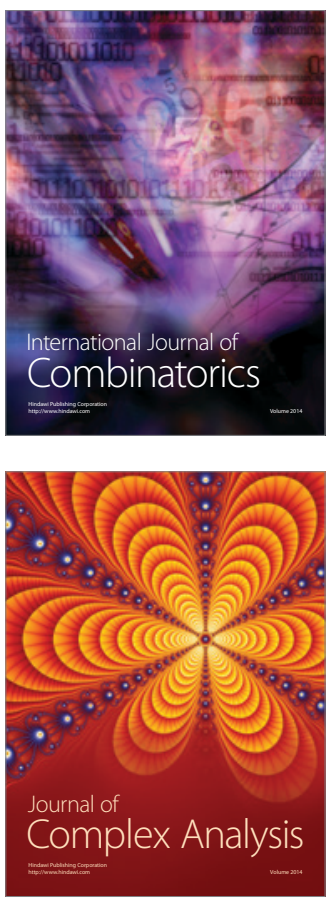

International Journal of

Mathematics and

Mathematical

Sciences
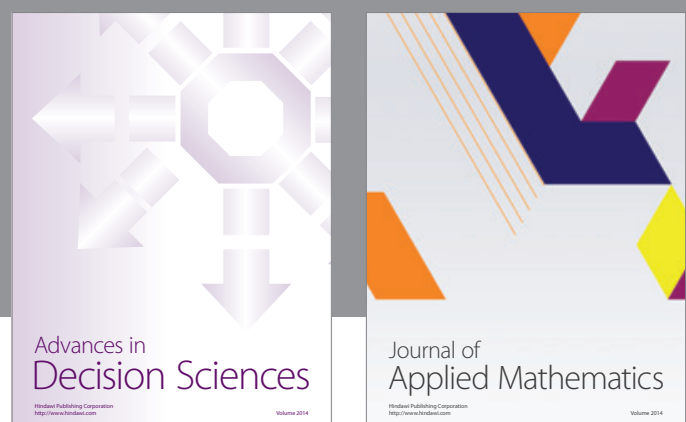

Journal of

Applied Mathematics
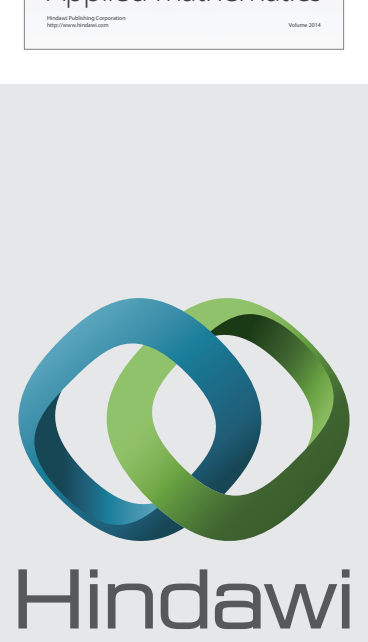

Submit your manuscripts at http://www.hindawi.com
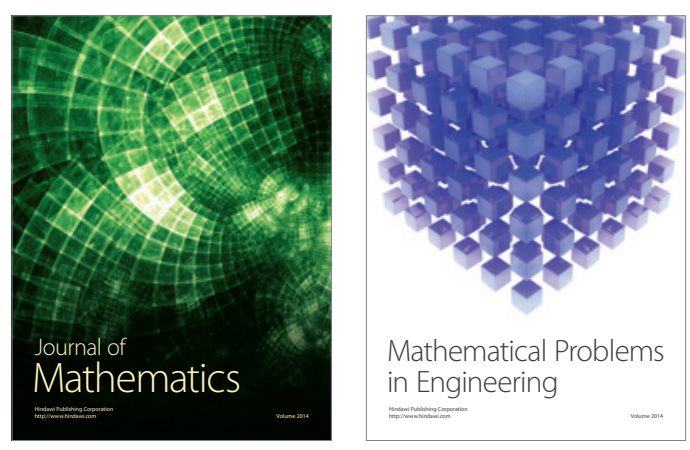

Mathematical Problems in Engineering
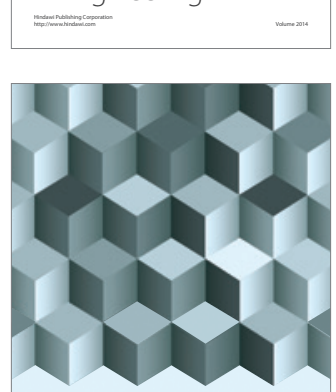

Journal of

Function Spaces
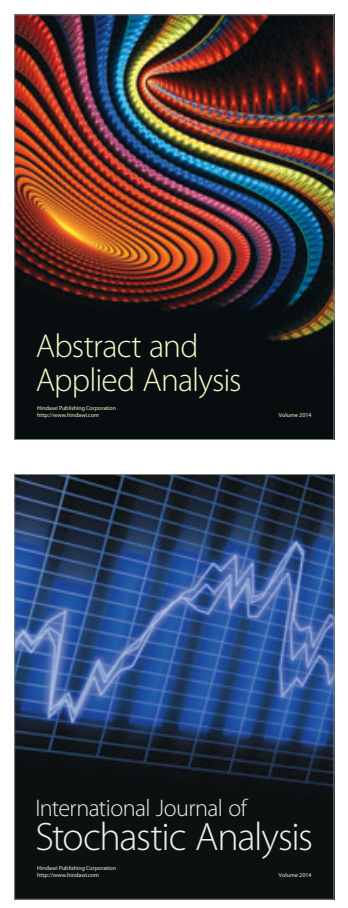

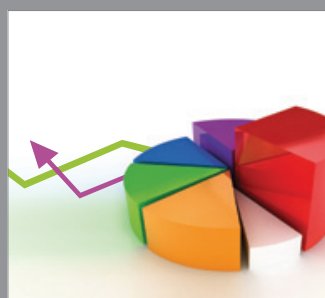

ournal of

Probability and Statistics

Promensencen
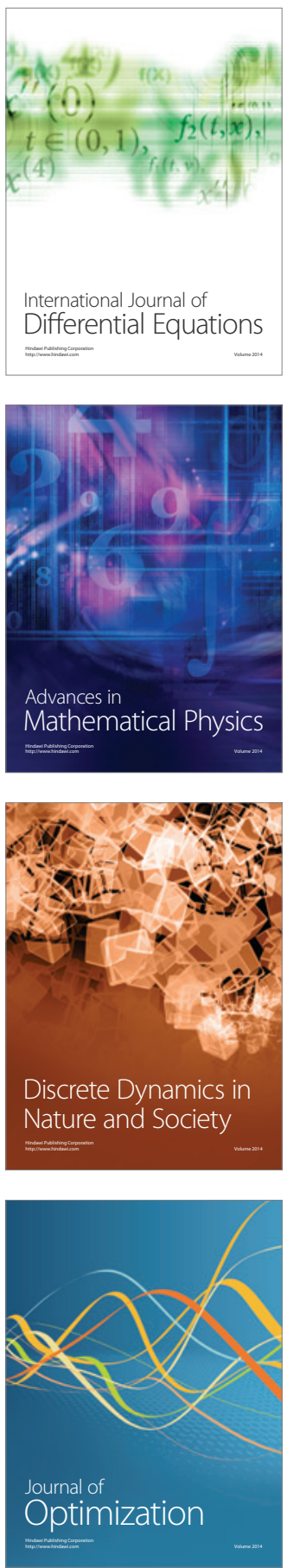